\title{
ENDOMETRIOSIS OF THE PLEURA
}

\author{
BY \\ HOWARD NICHOLSON \\ From University College Hospital, London \\ (RECEIVED FOR PUBLICATION SEPTEMBER 14, 1950)
}

Endometriosis may be defined as the growth of endometrium in situations other than the uterine cavity. The sites where these aberrant growths are most commonly found are the uterine wall, the ovaries, and the peritoneum of the pouch of Douglas. Cases are also recorded in which endometrial deposits were found in tissues remote from the genital tract, such as the bowel, the umbilicus, the bladder, and the ureters (Macleod, 1946). In the following case endometrial tissue was found in the visceral pleura, a finding which, so far as I know, has not been recorded previously.

\section{CASE REPORT}

A Ceylonese woman, aged 25, was admitted to University College Hospital on October 22, 1949. She had been well until the summer of 1948 , when she had developed pain over the right side of her chest. She did not feel ill at this time, but was said to be febrile. She was seen by her doctor in Colombo, and a radiograph of her chest was taken in October of that year (Fig. 1). She was advised that an aspiration of her right pleural cavity should be carried out, but she and her family were unwilling to allow this to be done. She went to the country, where she rested and remained fairly well until April, 1949. In that month she became very breathless on the slightest exertion, developed a pain in the right side of her abdomen, and became febrile to $102^{\circ} \mathrm{F}$. She was then seen again by her doctor, and a radiograph of her chest showed further displacement of her heart to the left by what appeared to be a massive pleural effusion on the right side.

Paracentesis of the right pleural cavity was carried out on April 12, 1949, when $80 \mathrm{oz}$. of heavily blood-stained liquid were aspirated, and air introduced in its place. A radiograph on the following day showed an extensive right pneumothorax with a basal

Fig. 1.-Radiograph taken in Ceylon, October 1, 1948.

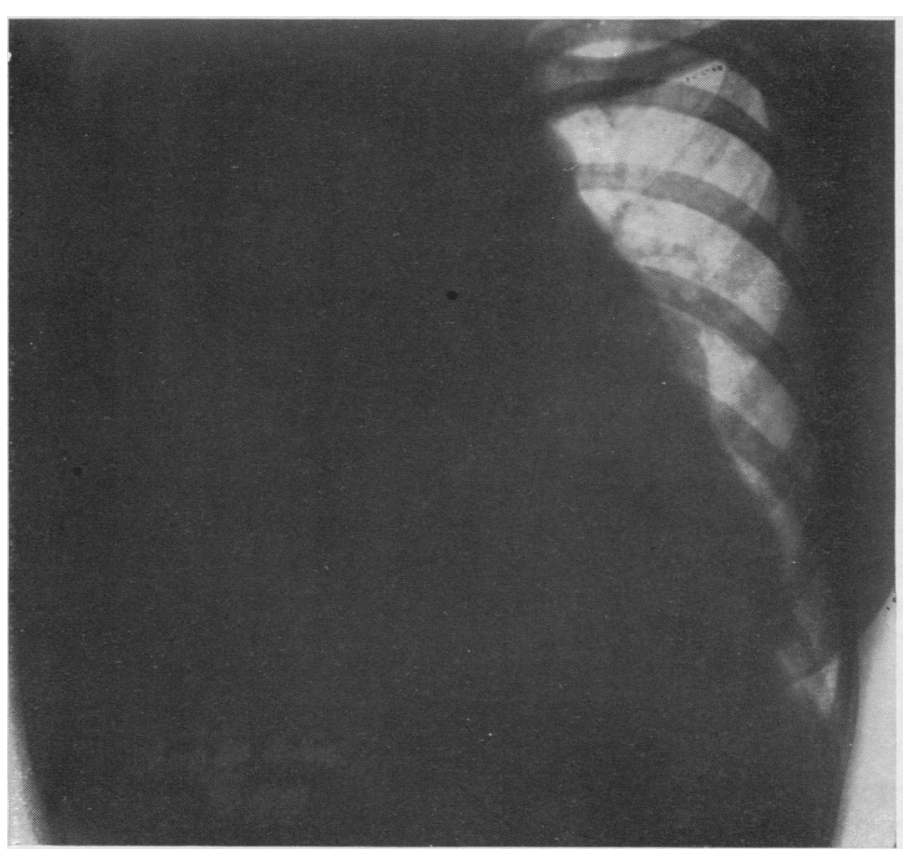



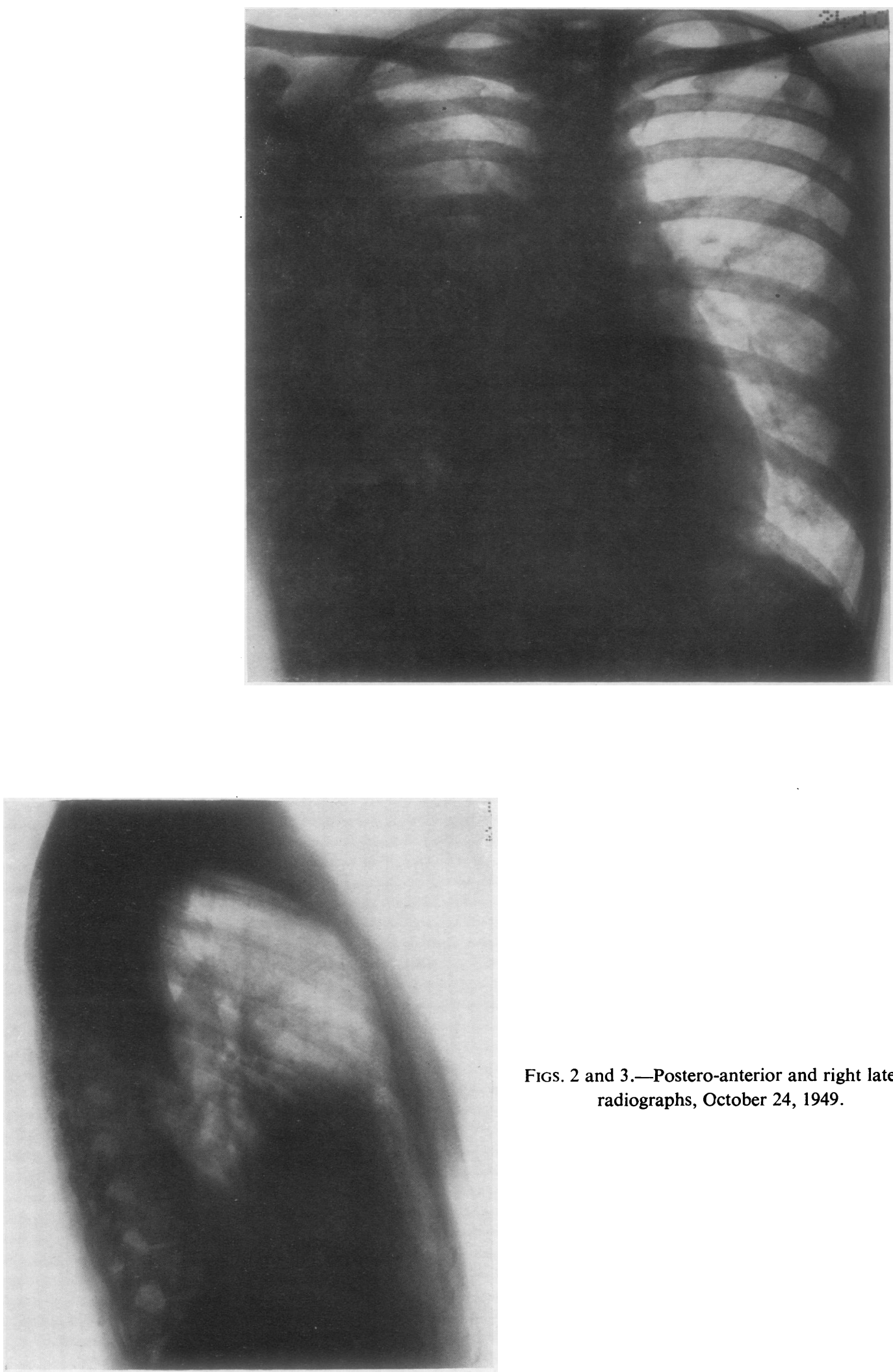

FIGS. 2 and 3.-Postero-anterior and right laterain radiographs, October 24, 1949. 


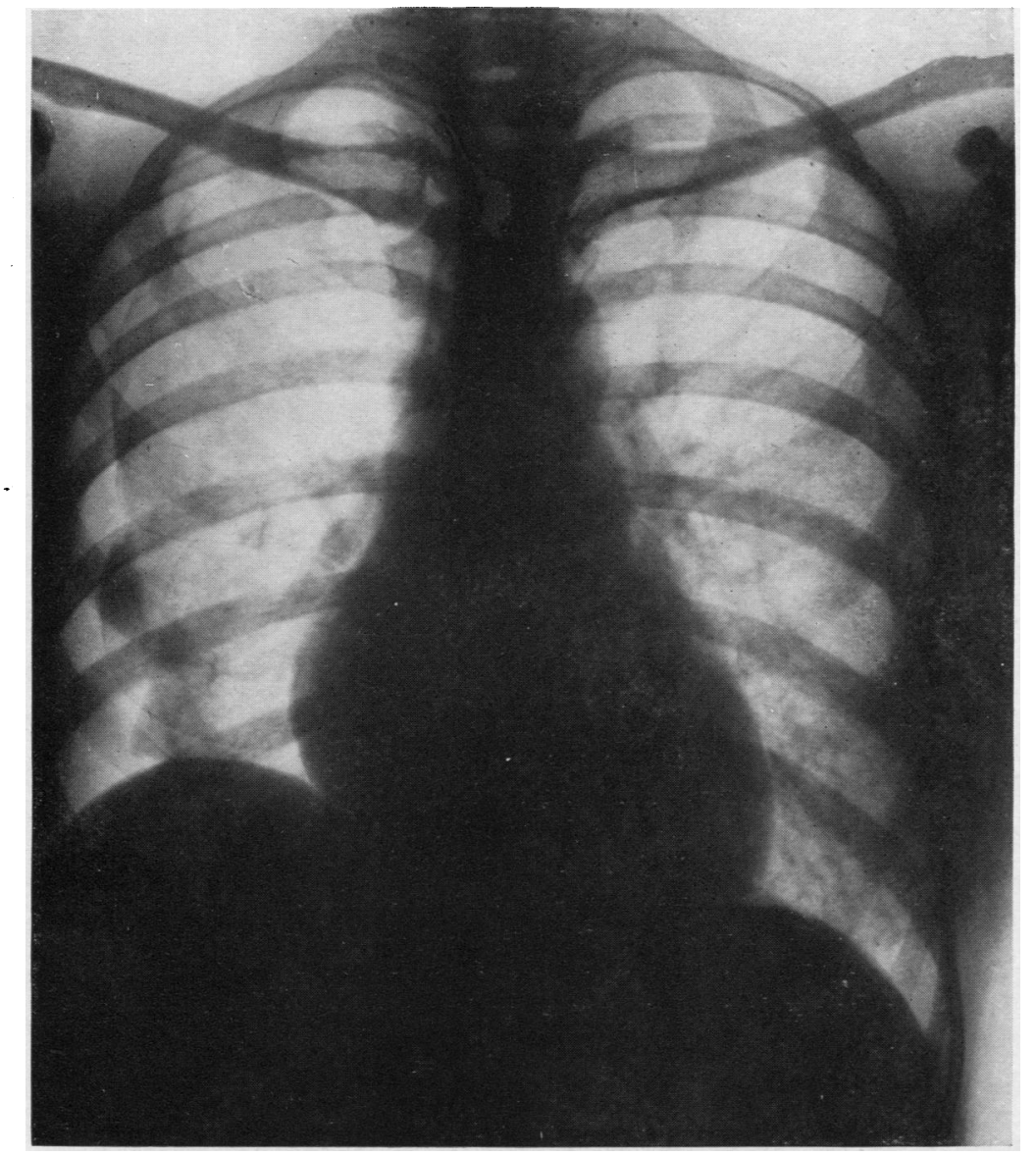

FiG. 4.-Postero-anterior radiograph taken after aspiration and air replacement, November 2, 1949.

FIG. 5.-Tomogram (7 cm. section), November 2, 1949.

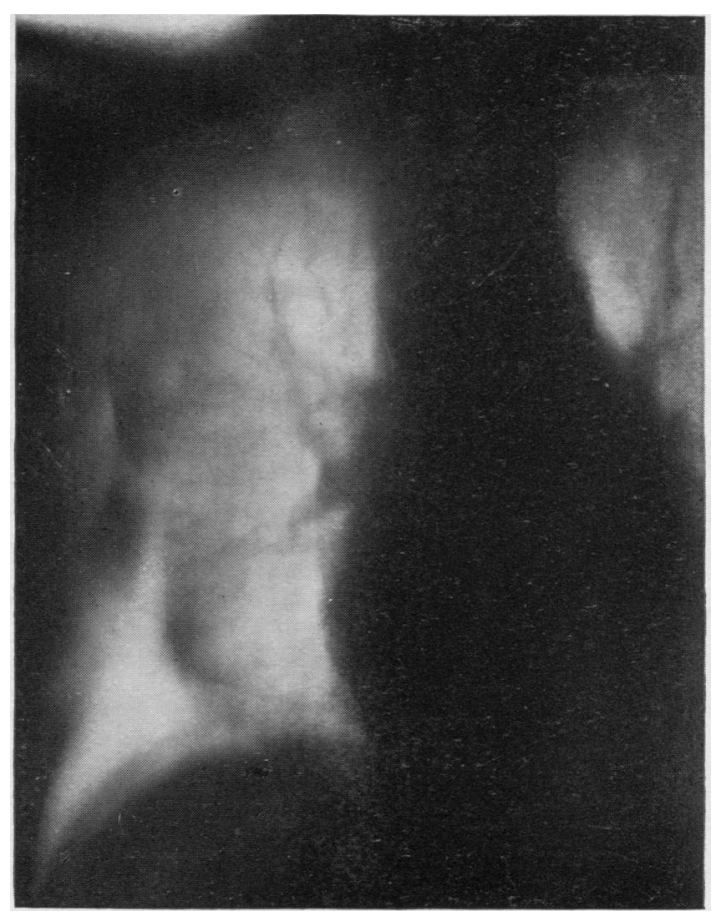


fluid-level. At this time the patient was still dyspnoeic and needed constant oxygen. Two days later $10 \mathrm{oz}$. of similar fluid were aspirated. During May the patient's general condition steadily improved, and a radiograph taken on June 6, 1949, showed some aeration of the right lung. The mediastinum was central, but there was still a large effusion on the right side with a fluid level at the first costal cartilage. In August the patient had improved sufficiently to be allowed to go once more into the country, but at the beginning of September she again developed pain over the right side of the chest and returned to the care of her doctor. A radiograph showed that the effusion had increased and displaced the mediastinum. Aspiration was carried out, and the fluid obtained was, as on the previous occasions, heavily blood-stained. The pathologist's report stated that it contained $5.3 \mathrm{~g}$. \% protein, numerous red and some white cells, as well as endothelial cells. No organisms of any sort were found. The aspiration was repeated on three occasions, after which the patient was sent to England by sea.

On her admission to University College Hospital she was in good general condition, afebrile, and not breathless on moderate exertion. She had no cough, no sputum, and no pain in her chest. She was small but not wasted. There was no finger clubbing. Abnormal physical signs were confined to her chest, where there was evidence of a right pleural effusion without displacement. of the mediastinum. The chest radiograph (Figs. 2 and 3) confirmed the presence of the effusion and showed a good deal of aerated lung on the right side. There was also a small amount of air in the right pleural cavity, no doubt remaining from the previous aspirations. A blood count showed $108 \%$ haemoglobin and 5,400,000 red cells per c.mm.; the total white cell count was 9,100 leucocytes per c.mm. with a normal differential. The erythrocyte sedimentation rate was $10 \mathrm{~mm}$. in one hour by the Westergren method. Bleeding time, clotting time, and platelet counts were normal.

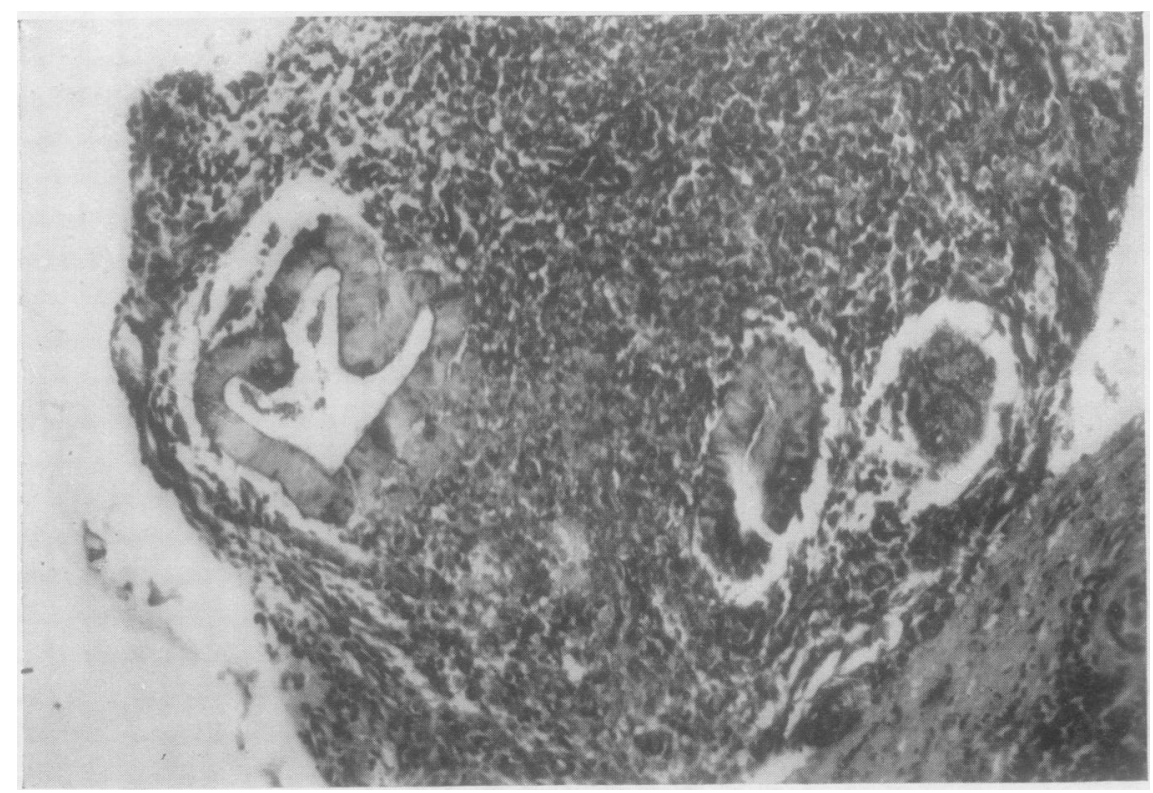

FIG. 6.-Photomicrograph of a section of the membrane dissected from the lung surface. Haematoxylin and eosin. $\times 50$. 

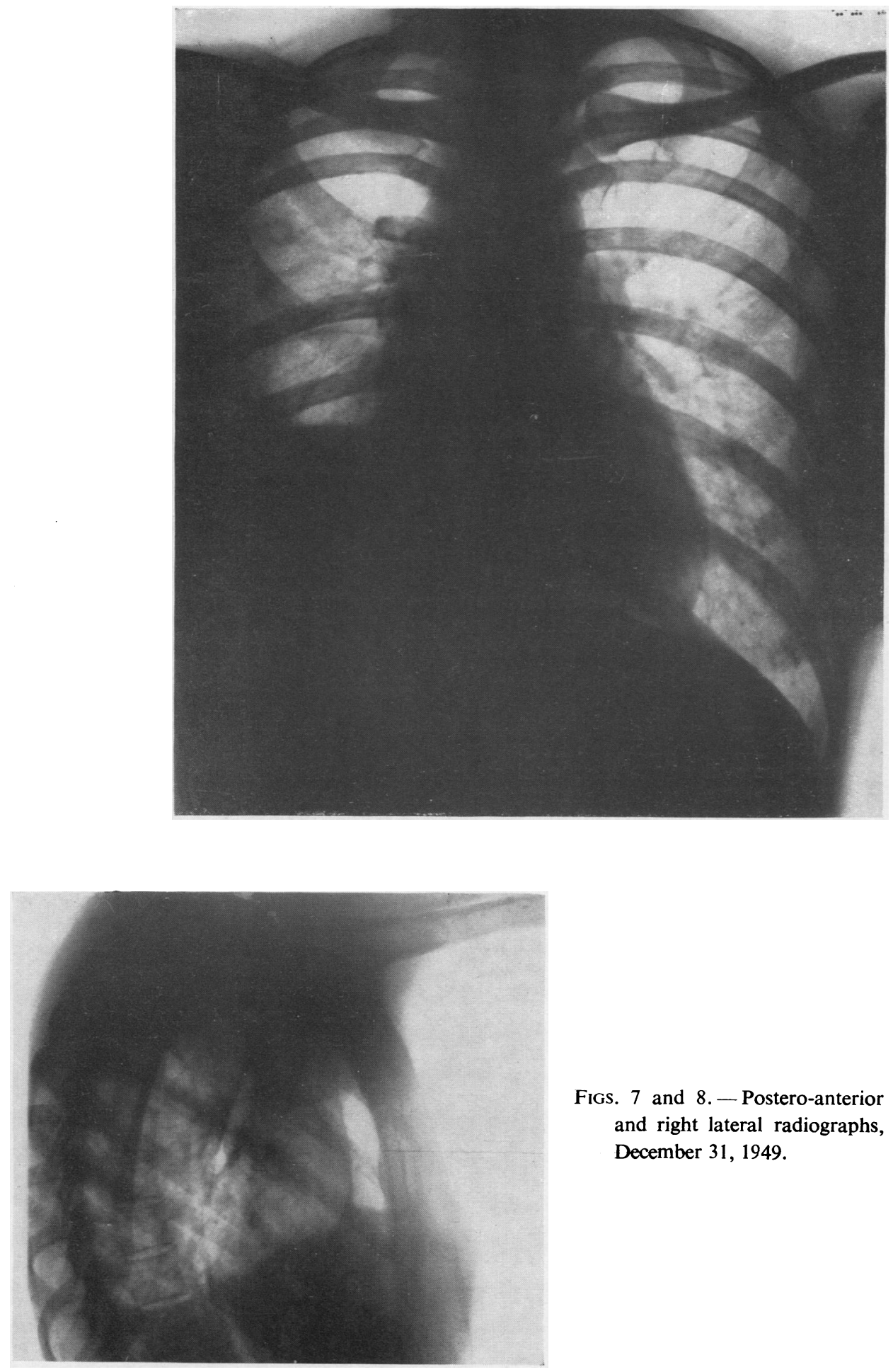

Figs. 7 and 8.- Postero-anterior and right lateral radiographs, December 31, 1949. 
On October 28, 1949, 25 oz. of apparently heavily blood-stained fluid were aspirated from her right chest. This and other specimens were found to contain numerous red and white cells. No organisms were seen in smears, and cultures were sterile after 48 hours' incubation. Cultures for the tubercle bacillus were also set up and remained sterile. Although the liquid aspirated was dark red in colour, its haemoglobin content was found to be $10 \%$. Aspiration with air-replacement was carried out on three successive days to empty as nearly as possible the pleural cavity. The radiograph (Fig. 4) taken afterwards showed a small pneumothorax and no obvious abnormality in the right lung. It was remarkable that, although the right pleural effusion had been known to be present for 18 months, the pleural layers did not seem to be thickened. Tomograms (Fig. 5 shows the $7 \mathrm{~cm}$. section) confirmed that there was little pleural thickening, but suggested that there might be a solid lesion or an area of atelectasis laterally in the middle lobe.

On November 15 thoracotomy was performed under general anaesthesia. The surface of the lung was covered by a thin, shining pellicle which in places had folded and puckered the upper and middle lobes. Over the middle lobe this thickened to form an opaque, yellow area about an inch across. No other abnormality was found and the diaphragm seemed normal. Because this pellicle appeared to be restricting the complete expansion of the lung it was stripped from the surface of the middle and most of the upper lobes. When the opaque, yellow patch was dissected from the lung it seemed to contain blood clot. The chest was closed, apical and basal intercostal catheters being inserted into the pleural cavity and attached to a water-seal bottle. After 24 hours these catheters were attached to a suction pump in an attempt to produce complete re-expansion of the lung. There was a good deal of alveolar leakage of air, and this attempt was not successful ; although the lung remained aerated, complete re-expansion was not obtained. When the catheters were removed after eight days there was still a small hydropneumothorax.

Pieces of the membrane dissected from the lung surface were examined histologically. A section is shown in Fig. 6. The presence of typical endometrium can be seen.

After removal of the intercostal catheters, the patient was allowed to get up and soon felt well. A gynaecological investigation did not reveal any evidence of pelvic endometriosis. Her menstrual history was normal, menstruation having begun at the age of 15. She was married in August, 1948, but had not been pregnant. In retrospect it did not seem certain that her chest pain was related to her menstrual periods.

In view of her youth it was not thought justifiable to advise her to have an artificial menopause, and testosterone tablets were implanted subcutaneously with the idea of reducing endometrial activity. She returned to Ceylon early in January, 1950, and radiographs were made on December 31, 1949 (Figs. 7 and 8). Her chest had not been aspirated during the preceding month and there had been little accumulation of fluid. She was advised to become pregnant, since endometriosis may disappear during pregnancy (Macleod, 1946). She has remained well in Ceylon, but has not become pregnant.

Since this paper was submitted for publication the following information has been received. In January, 1951, the patient once more had pain in the right chest and was febrile and breathless. On aspiration $16 \mathrm{oz}$. of blood-stained fluid were obtained from the right pleural cavity. Oophorectomy was carried out in India and endometrial deposits were seen on the pelvic peritoneum.

\section{Discussion}

While this case is recorded as an extreme rarity, it is perhaps worth while to draw attention to a feature which might have suggested the diagnosis. In spite of there having been haemorrhagic fluid present in the right pleural cavity for more than 
18 months, the pleural layers, as shown radiologically, were clearly not covered by deposited fibrin. The only sort of haemorrhagic fluid which does not contain fibrin is menstrual blood.*

My thanks are due to Dr. Byron Josef, who treated the patient in Colombo; to Mr. C. Price Thomas, who operated on her; and to Dr. J. C. Clegg for the section shown in Fig. 6.

\section{REFERENCE}

Macleod, D. (1946). Brit. J. Surg., 34, 109.

* This case is being reported elsewhere, and its implications in relation to the pathogenesis of endometriosis considered, by Miss J. Barnes, F.R.C.S., F.R.C.O.G. 